\title{
Hippocampal and Neocortical Interactions During Context Discrimination: Electrophysiological Evidence From the Rat
}

\author{
Sheri J.Y. Mizumori, ${ }^{1 *}$ David M. Smith, ${ }^{2}$ and Corey B. Puryear ${ }^{1}$
}

\begin{abstract}
There is substantial evidence that hippocampus plays an important role in the processing of contextual information. Its specific role, however, remains unclear. One possibility is that single hippocampal neurons represent context information so that local circuits can construct representations of the current context, and the context that is expected based on past experience. Population codes derived from input by multiple local circuits may then engage match-mismatch algorithms that compare current and expected context information to determine the extent to which an expected context has changed. The results of such match-mismatch comparisons can be used to discriminate contexts. When context changes are detected, efferent messages may be passed on to connected neocortical areas so that informed "decisions" regarding future behavioral and cognitive strategies can be made. Here, a brief review describes evidence that a primary consequence of hippocampal processing is the discrimination of meaningful contexts. Then, the functional significance of neocortical circuits that likely receive hippocampal output messages are described in terms of their contribution to the control of ongoing behavioral and cognitive strategy, especially during active navigation. It is clear from this systems view that studies of spatial navigation continue to provide researchers with an excellent model of hippocampal-neocortical interactions during learning. $\odot 2007$ Wiley-Liss, Inc.
\end{abstract}

KEY WORDS: context; place cells; spatial processing; temporal processing; behavioral intention

\section{INTRODUCTION}

In recent years, an increasingly impressive array of methodologies and perspectives has allowed us to gain new, and sometimes surprising, insight into the dynamic (intrinsic) neural operations and behavioral consequences of hippocampal function. Converging evidence has led to the general conclusion that hippocampus is essential for context processing, especially as it relates to episodic memory function (e.g. O'Keefe and Nadel, 1978; Nadel and Wilner, 1980; Nadel and Payne, 2002; Tulving, 2002). It has become equally clear that fully adaptive context analysis and learning requires not only hippocampus, but the larger neural system within which hippocampus operates. In what follows, it is argued that the specific contribution that hippocampus makes to context processing is to detect changes in context so that connected areas (especially neocortex) can make

\footnotetext{
${ }^{1}$ Department of Psychology, University of Washington, Seattle, Washington; ${ }^{2}$ Department of Psychology, Cornell University, Ithaca, New York

Grant sponsor: NIMH; Grant number: 58755.

*Correspondence to: Sheri J. Y. Mizumori, Psychology Department, University of Washington, Box 351525, Seattle, WA 98195-1525, USA.

E-mail: mizumori@u.washington.edu

Accepted for publication 1 May 2007

DOI 10.1002/hipo.20317

Published online 27 June 2007 in Wiley InterScience (www.interscience. wiley.com).
}

informed "decisions" in terms of future behavioral and cognitive strategy. First, a brief review is provided concerning the role for hippocampus in context discrimination. Then, the function significance of neocortical circuits that likely receive hippocampal output messages are described in terms of their contribution to the control of ongoing behavior and cognitive strategy.

\section{HIPPOCAMPUS IS ESSENTIAL FOR CONTEXT PROCESSING}

There is now strong evidence that hippocampus processes contextual information (e.g. Hirsh, 1974; Myers and Gluck, 1994; Anagnostaras et al., 2001; Maren, 2001; Fanselow and Poulos, 2005; Bouton et al., 2006). Conditioned fear responses to contextual stimuli is eliminated with hippocampal lesions even though responses to discrete conditional stimuli remain intact (Kim and Fanselow, 1992; Phillips and LeDoux, 1992, 1994). Also, hippocampal or entorhinal cortical damage produces an insensitivity to changes in context as evidenced by the fact that lesioned animals do not show the normal decrement in conditioned responding when the context is altered (Penick and Solomon, 1991; Freeman et al., 1996a,b). Manipulations that impact hippocampal synaptic plasticity (e.g. LTP) also affect context learning (e.g. Shors and Matzel, 1997). These results not only support the hypothesis that hippocampus processes context information, but more specifically, they indicate that hippocampus plays a particularly salient role in the discrimination of meaningful contexts (Smith and Mizumori, 2006a).

A context processing account of hippocampus is consistent with hypotheses that hippocampus plays a special role in the flexible use of conjunctive, sequential, relational, and spatial information (e.g. O'Keefe and Nadel, 1978; Foster et al., 1987; Eichenbaum et al., 1999; Wood et al., 2000; Eichenbaum and Cohen, 2001; O'Reilly and Rudy, 2001; Fortin et al., 2002). These flexible, spatial, sequential, and relational operations likely enable hippocampus to make accurate context discriminations. While significant evidence supporting the existence of these operations in hippocampus can be derived from single unit recordings in behaving rats, it remains a significant challenge to understand how context processing theories of hippocampus account for the varied hippocampal place cell 
responses that have been reported. The following description of a context discrimination hypothesis $(\mathrm{CDH}$; Smith and Mizumori, 2006a; Mizumori, 2007) provides a theoretical framework to account for a significant amount of the place cell findings, as well as to provide a link to current ideas about the specific role of the hippocampus in learning and episodic memory (Tulving, 2002). While many features of this hypothesis build on concepts discussed by other investigators (as pointed out below), its consideration here provides an opportunity to elaborate specifically on the functional networks that should exist as part of hippocampal neural organization. Furthermore, the $\mathrm{CDH}$ makes clear predictions about how hippocampal efferent messages ultimately come to impact ongoing behavior.

Consistent with a large body of electrophysiological evidence, $\mathrm{CDH}$ postulates that single hippocampal neuronal representations of context provide data to population-based network computations that ultimately determine whether expected contextual features of a situation have changed (e.g. Mizumori et al., 1999b, 2000, in press; Smith and Mizumori, 2006a,b; Mizumori, 2007). That is, hippocampal representations of multiple aspects of the current context (Fig. 1; O'Keefe and Nadel, 1978; Nadel and Wilner, 1980; Nadel and Payne, 2002) may contribute to a match-mismatch type of analysis that evaluates the present context according to how similar it is to the context that an animal is expecting based on past experience (e.g. Gray, 1982; Vinogradova, 1995; Mizumori et al., 1999b, 2000; Gray, 2000; Lisman and Otmakhova, 2001; Anderson and Jeffery, 2003; Hasselmo et al., 2002; Jeffery et al., 2004; Hasselmo, 2005; Manns et al., 2007). Detected mismatches can be used to identify novel situations and to distinguish different contexts, functions that are necessary to define significant events or episodes. When a match is computed, the effect of hippocampal output could be to strengthen currently active memory networks located elsewhere in the brain (e.g. neocortex). In this way, hippocampus potentially plays different mnemonic roles depending on whether or not contexts actually change.

The detection of changes in context is fundamentally important for accurate performance in a variety of learning tasks (e.g. navigation-based learning, instrumental conditioning, classical conditioning). This function is critical because of the need to engage cellular mechanisms for new learning at potentially important times (Paulsen and Moser, 1998). Indeed, Smith and Mizumori (2006b) showed that hippocampal neurons develop context-specific responses, but only when rats were required to discriminate contexts. Discriminating neural responses were not observed when rats were allowed to randomly forage for the same amount of time. Most recently, Manns et al. (2007) showed that relative to match trials in an odor cue or object recognition task, CA1 neurons preferentially discharged when animals experienced a nonmatch situation in these same tasks. Moreover, cell firing tended to occur during the "encoding phase" of the ongoing theta rhythm. This interpretation is based on the hypothesis that encoding and retrieval alternates at a frequency that coincides with the theta frequency (see review in Hasselmo, 2005). Thus, detection of a nonmatch situation can change the relationship between cell discharge and the local theta rhythm such that encoding functions
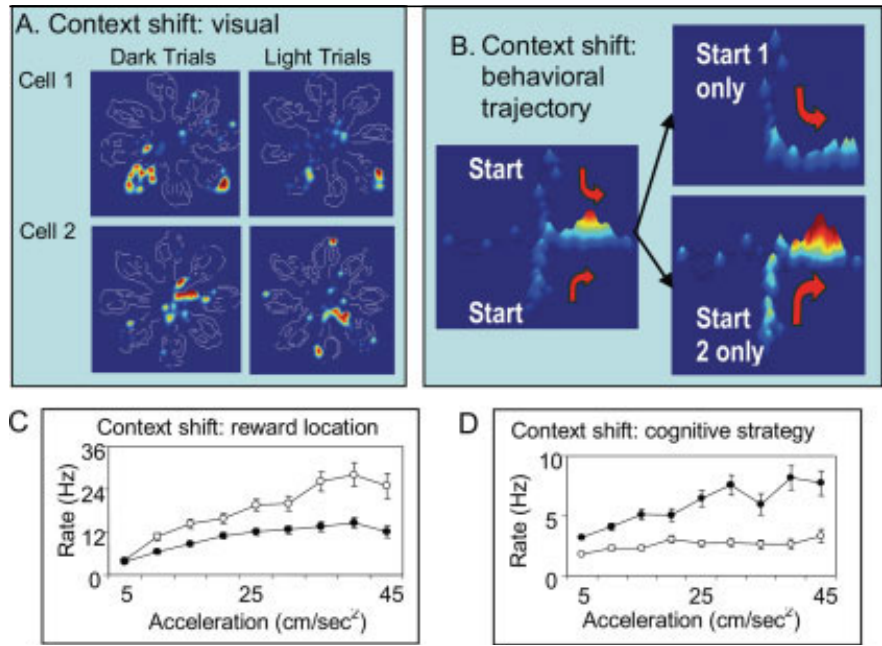

FIGURE 1. Color density plots of spatially localized firing show context-dependent changes in place fields (Panels $A$ and $B$ ) and egocentric movement correlates of interneurons (Panels $C$ and D). Panel A shows place field reorganization when the room light conditions change as rats perform the same spatial memory task during both dark and light phases of testing on a radial maze. Such responses are almost always observed when the recording session begins in darkness. Presumably this place field reorganization occurs because the appropriate memory has not yet been recalled. When the lights are turned on, a different (learned) representation appears, and these have been shown to remain stable during subsequent dark periods (e.g. Mizumori et al., 1999b). Panel B demonstrates that the exhibition of a given place field may be conditional depending upon the recent behavioral history of the animal. In this case, place fields were recorded as rats started a plus maze task from either north (Start 1) or south (Start 2) maze arms to obtain food located on the east (right) maze arm. Within session, data were divided according to the start arm location. It was found that the place field was observed only when the rat began a trial from the south location. Importantly, this was the case even though the rat exhibited the same behaviors, and experienced the same external sensory environment as it traversed the place field location. (Panels C and D) Hippocampal interneurons often vary firing rates as a function of the velocity or acceleration of translational movements through extended space (e.g. McNaughton et al., 1983). Panel C (left) illustrates such movement-related firing (open circles) when a rat performed a plus maze task similar to the one described in Panel B. When the reward location changed from the east to west maze arm, it can be seen that the acceleration-correlated firing was sharply attenuated (closed circles) even though the rat continued to engage in the same type of behavior as before the reward location shift (Smith and Mizumori, 2006). Panel $D$ illustrates the effects of changes in cognitive strategy (from spatial to response strategies) on acceleration correlates of a hippocampal interneuron. This cell initially did not show significant acceleration correlated firing. However, after the strategy switch, an acceleration correlate emerged (Eschenko and Mizumori, 2007). Importantly, such changes in movement-correlated firing were observed for many cells even though the sensory and behavioral responses of the animal did not change. Panels $C$ and $D$, then, illustrate that interneurons represent context-dependent movement information. [Color figure can be viewed in the online issue, which is available at www.interscience.wiley.com.]

are enhanced. Detection of matches, on the other hand, does not cause changes in the hippocampal neural activity profile, resulting in efferent messages that continue to retrieve/utilize the currently active memory network. 
In further support of the $\mathrm{CDH}$, disconnecting hippocampus by fornix lesions impairs context discrimination (Smith et al., 2004), and hippocampal lesions reduce animals' ability to respond to changes in a familiar environment (Good and Honey, 1991; Save et al., 1992a,b). Spatial novelty detection corresponds to selective elevation of the immediate early gene c-fos in hippocampus, and not in surrounding parahippocampal cortical regions (Jenkins et al., 2004). Also, hippocampal neurons show significantly altered firing patterns when rats experience spatial or nonspatial changes in a familiar environment (O'Keefe, 1976; Muller and Kubie, 1987; Wood et al., 1999; Fyhn et al., 2002; Ferbinteanu and Shapiro, 2003; Moita et al., 2004; Yeshenko et al., 2004; Leutgeb JK et al., 2005; Leutgeb S et al., 2005; Puryear et al., 2006; Smith and Mizumori, 2006b; Eschenko and Mizumori, 2007).

The specific patterns of neural change after altering different features of an experimental context tend to be multidimensional

\section{Context Matrix}

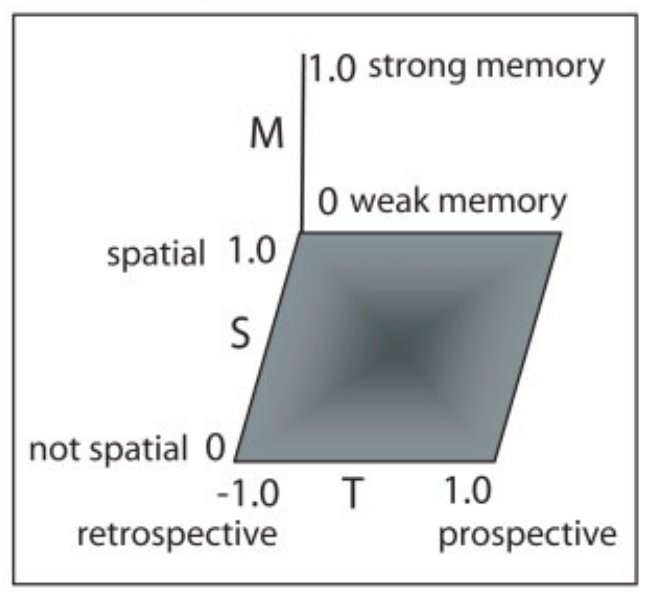

\section{Level 1 Integration: Place Cell Code}

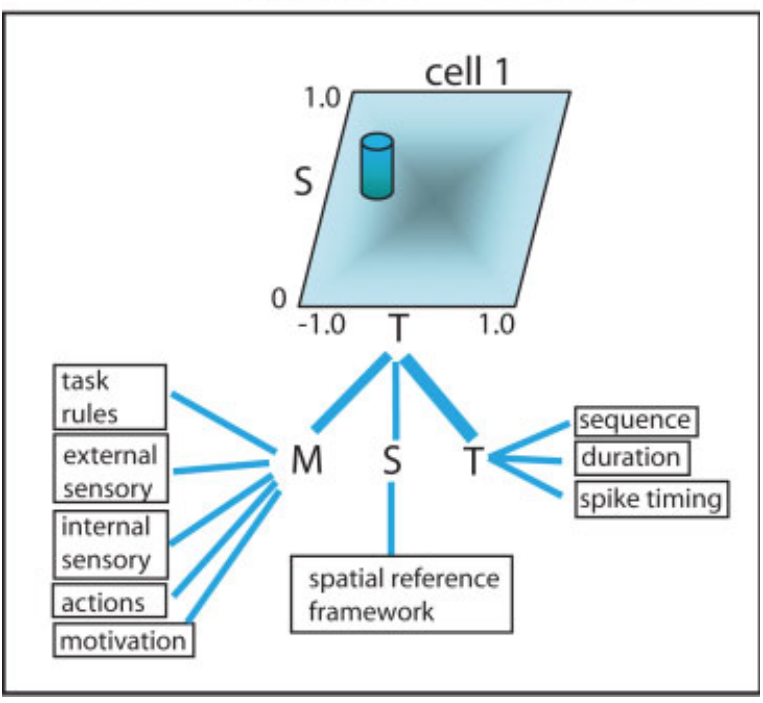

and complex (see Special issue on Place Fields and Episodic Memory, vol. 16(9), Hippocampus). After considering the diversity of neural responses and test conditions that have been reported, it appears that there are (at least) three qualitatively distinct types of influences on hippocampal neural codes that likely occur automatically regardless of the specific task demands: memory (i.e. past experience), spatial, and temporal (Fig. 2). This proposal is based in part on past suggestions that hippocampal-dependent memory involves associations of spatial, and temporal features (e.g. O'Keefe and Nadel, 1978; Mizumori et al., 2000; Redish et al., 2000; Burgess et al., 2001; Eichenbaum and Cohen, 2001; Morris, 2001; O'Reilly and Rudy, 2001; Buzsáki, 2005). At slight variance with many of these proposals, Mizumori (2007) suggest that the default mode of hippocampal processing is to continually integrate memory-guided perceptions of sensory, movement, and motivational information, or memory $(\mathrm{M})$, within a spatial $(\mathrm{S})$ reference framework as a function of time (T; Fig. 2). An important point here is that hippocampus may receive highly preprocessed $\mathrm{M}$ information so that it can become incorporated into specific spatial and temporal frameworks. The integration is proposed to occur according to a hierarchically organized scheme that incorporates $\mathrm{M}, \mathrm{S}$, and $\mathrm{T}$ information as fundamental inputs. As a result, during unrestrained navigation, place fields appear as neural representations of

FIGURE 2. Place cells are known to be responsive to multiple types of information. The context discrimination hypothesis postulates that hippocampus receives highly preprocessed information that reflects a perception of sensory (external and internal information), appropriate actions, and task rules according to past experience (or Memory, M). $M$ varies from 0.0 to 1.0 along the $z$-axis of the context matrix shown at the top. This range reflects zero to significant influences of past experience, respectively. As animals explore their environment, entorhinal cortex grid cells automatically provide hippocampus with a spatial reference frame $(S)$ within which contextual information can be placed. This feature could account for the consistent finding of place fields during navigation of spatial and nonspatial tasks (Eschenko and Mizumori, 2007). With training, however, the strength of $S$ can be made to vary from 0.0 to 1.0 to reflect weak to strong spatial organization. A third organizational influence on place field codes is temporal $(T)$ in nature. $T$ factors organize spike firing relative to the firing of other cells, salient stimuli, or behavioral responses. As a result, hippocampal neurons appear to code sequences of sensory and response information, and relay information about stimulus or response duration. Furthermore, many studies have shown that place cells fire relative to future or past events, and that place fields can be found to correspond to all locations within an environment. Therefore, $\mathrm{CDH}$ suggests that hippocampal neural representations can be aligned along a gradient that reflects the extent to which the context code represents past (retrospective) events or future (expected, or prospective) events. The location of the peak of the cylinder within the threedimensional matrix identifies for each cell the relative contributions of $M, S$, and $T$ input. The width of the lines reflects the relative strengths of the different input. The cell illustrated shows strong retrospective $(T)$ and $M$ components and a moderately strong $S$ influence. [Color figure can be viewed in the online issue, which is available at www.interscience.wiley.com.] 


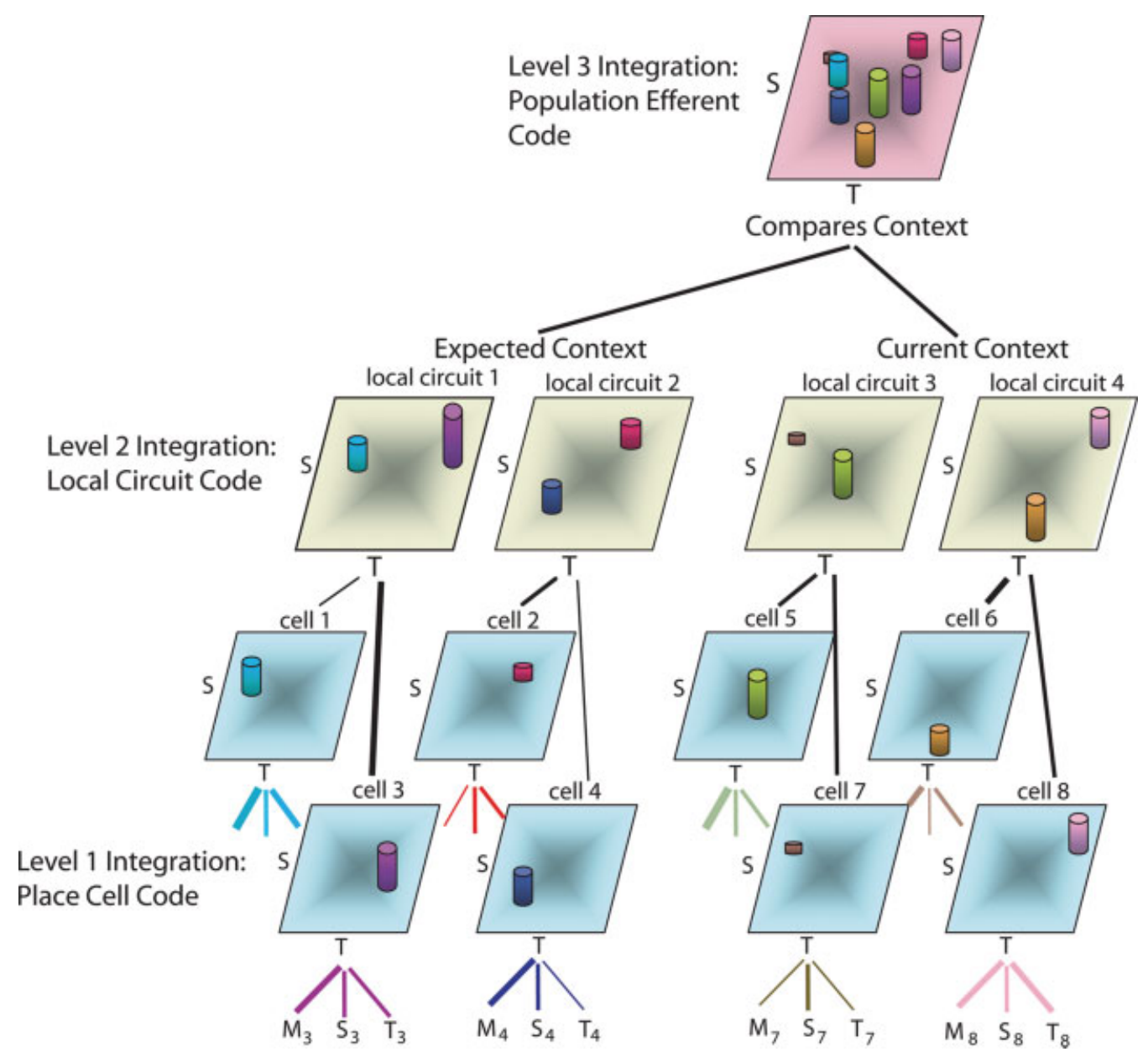

FIGURE 3. A hierarchical representation of context processing within hippocampus. Each square corresponds to a context matrix that reflects multiple types of information coded by single cells (Level 1; light blue), local ensembles (Level 2; tan), or large regions of hippocampus (Level 3; pink). The width of the lines reflects the relative strengths of the different input. Subscripts correspond to the $M, S$, or $T$ input for a given cell number. Place cell studies reveal that a number of specific features (e.g. sensory, behavioral, motivation, and knowledge of task rules; see Fig. 2) are represented in hippocampal networks according to past experience or memory $(M)$. The $M$ input to place cells may vary in strength depending on the learned significance of the information that $M$ represents. As a result, some $M$ inputs may disproportionately present current context information to hippocampal networks, while other $M$ inputs may be influenced more by expectations for a given context, such as memory for task rules. Another factor that continually shapes the organization of information in hippocampus is the spatial reference framework $(S)$ provided by entorhinal cortex grid cells (described in text). A third continual influence on hippocampal place fields is one that strives to organize incoming information as a function of time $(T)$, both retrospectively and prospectively. Level 2 neural integration reflects ensemble activity of local networks of cells. Presumably, this level integrates representations by single place cells to define portions of the expected and current context within local ensembles. In this example, Cells 1 and 3 combine to define a part of what will become a component of the definition of the expected features of a context. Cell 3 has stronger influence than Cell 2, perhaps reflecting a stronger history of synaptic activation. Cells 2 and 4 similarly combine to define another portion of the expected context. Cells 5 and 7 combine to define a segment of the current context. The greater influence at Level 2 is depicted by the taller cylinder. Finally, Cells 6 and 8 combine to form another local ensemble that processes current context information. Cell 6 has greater influence on Level 2 integration, and this is shown by the taller cylinder in Local circuit 4 . Presumably there are many such local circuits throughout hippocampus, and many more than two individual place cells contribute to each local ensemble. Also, due to the known massive interconnection between cells in a specific hippocampal subregion, it is likely that the activity of one ensemble impacts the activity of connected ensembles (not shown). Level 3 integration compares expected and current context ensemble representations to produce an efferent code that indicates the extent to which the expected context has changed. If the expected and current contexts differ, as in the present case, a signal identifying the nonmatch will be forwarded to cortical systems to notify them of the change. This notification could have the effect of altering cell excitability patterns so that the nature of the change can be evaluated. An example of one such consequence could be an increase in exploratory behaviors. If the ensemble codes for the expected and current context are the same, the expected and current context matrices should overlap. In this case, the output message of hippocampus may signal the continuation of the ongoing behavior, and it may strengthen synaptic connections within the neural network that defines the current activated memory. The result of the pattern of activity shown in this figure exemplifies an output message during a nonmatch condition. [Color figure can be viewed in the online issue, which is available at www.interscience.wiley.com.] 


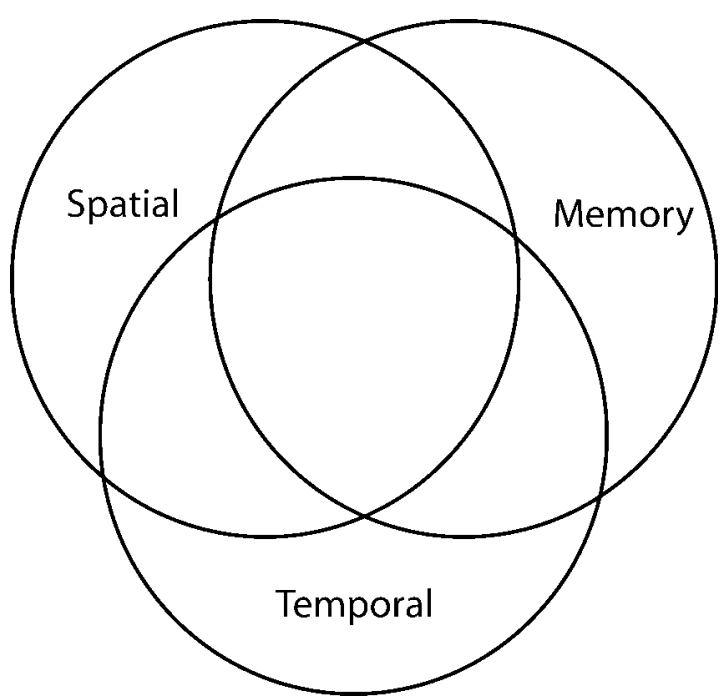

FIGURE 4. Schematic diagram illustrating the many relationships that likely exist between Memory (M), Spatial (S), and Temporal (T) inputs to hippocampal cells. Some inputs may occur independently from the others, while other inputs could reflect combinations of two or three influence types.

different types of sensory, behavioral, and intrinsic information that have strong spatial and temporal features, thereby validating the term spatial context when referring to the meaning of place field representation (Nadel et al., 1985; Mizumori et al., 1999b, 2000; Jeffery et al., 2004). CDH, then, suggests a more complex definition of context than is typically used in the learning literature-a definition that emphasizes the integration of sensory, motivational, response, and mnemonic input within a spatial framework as a function of time. That is, hippocampal context codes are not just a reflection of the external sensory environment.

HIERARCHICALLY ORGANIZED CONTEXT PROCESSING WITHIN HIPPOCAMPUS

Previous hierarchical models of hippocampal processing consider the fundamental elements of the hierarchy to be either stimulus-defining information (e.g. Shapiro et al., 1997) or individual events (e.g. Eichenbaum et al., 1999; Shapiro and Eichenbaum, 1999; Wood et al., 1999). Figure 3 illustrates an alternative hierarchical model that includes as inputs $S$ (spatial) information as a form of organization rather than specific environmental features that individual cells discriminate. Also, M (memory) input from extrahippocampal systems brings preselected task-relevant sensory, behavioral, motivational, and learned units of information into hippocampus. Presumably, such $\mathrm{M}$ input is derived from neocortical memory circuits. Finally, it is argued that the T (temporal) organization of information may not necessarily represent only the end goal of hippocampal processing. Rather it may also emerge from intrinsic synaptic plasticity mechanisms that can be guided by extrahippocampal temporal events (e.g. Buzsaki, 2005). These synaptic mechanisms that temporally organize salient information allow hippocampus to ultimately define expected and current contextual features in terms of their duration or sequence. Direct connections to CA1 from entorhinal cortex may provide $M$ inputs to hippocampus that aid in the segregation of sequences into meaningful epochs at multiple levels. It should be pointed out that $\mathrm{M}, \mathrm{S}$, and $\mathrm{T}$ information may not be mutually exclusive. Rather, there may be moderate to considerable interaction amongst these variables prior to and within hippocampus (Fig. 4). As an example, the reliability or specificity of a particular place field could be based on $M$ inputs. Indeed it has been shown that such measures of place fields vary with experience (e.g. Mizumori and Kalyani, 1997; Puryear et al., 2006). Also, the degree of temporal coherence amongst a group of neurons could depend on their recent activation patterns (e.g. Shapiro and Ferbinteanu, 2006). As a third example, spatial features could conceivably determine the degree of temporally correlated firing one could expect to observe between especially place cell pairs.

Consideration of the details and significance of place field representations within a hierarchically organized scheme suggests three basic levels of integration (Fig. 3; Mizumori, 2007). The basic features of each level are summarized in Table 1. Essentially, Level 1 allows for the initial formulation of the spatialtemporal context features that are typically observed for place fields. Level 2 integrates single neuron context representations into local circuit signals that reflect current context information or expected context information in experience-dependent ways. Local circuit neural codes could be reflected in the firing of one cell relative to the firing of another cell within the same circuit. Moreover, given that Level 2 integrates input from multiple individual neurons, correlated firing between cells may be found to preferentially occur relative to general task events such as the beginning and end of trials, as has been shown by Shapiro and Ferbinteanu (2006). Level 3 integration is considered to progressively carry out the context comparison function amongst an increasingly greater number of local circuit arrays, perhaps according to mechanisms such as pattern completion and pattern separation.

In contrast to $\mathrm{M}$ and $\mathrm{S}$ information (discussed in more detail in the following section), $\mathrm{T}$ information seems to be driven largely (although not exclusively) by circuitry intrinsic to hippocampus. This has been shown primarily from studies of how place cell firing is temporally regulated. This temporal organization may take different forms, perhaps for different purposes. On a single pass through a field, the first spike of successive bursts occurs at progressively earlier phases of the theta cycle (O'Keefe and Recce, 1993). It has been suggested that such temporally dynamic changes in spike-timing (called phase precession) may be a key mechanism by which place fields provide a link between temporally-extended behaviors of an animal and the comparatively rapid synaptic plasticity mechanisms (e.g. LTP) that are thought to subserve learning (e.g. Skaggs et al., 1996). Theoretical considerations have generated detailed models of how phase precession could explain the link between 
TABLE 1.

\section{Basic Features of the Proposed Tri-Level Organization Within Hippocampus}

Level 1 organization:

Place Cell Codes (a) Place cells receive highly preprocessed information in the form of input signals that reflect memory-guided perceptions (M) of external and internal states. Presumably, these inputs derive from neocortical memory network activity.

(b) Place cells organize $\mathrm{M}$ information within a spatial framework (S) provided by entorhinal cortex, a framework that reflects the current spatially-extended surround.

(c) Intrinsic and extrinsic hippocampal neural circuitry also organize $\mathrm{M}$ information as a function of time (T) by regulating spike timing relative to sensory stimulation, the discharge of other cells, or the EEG. Such temporal processing likely underlies sequence coding. It also leads to the generation of predictions for future behaviors (a prospective code) and a retrospective code for recent behaviors.

Level 2 organization: Local Circuit Codes

(a) Local neural circuits code integrate context information from individual place cells in preparation, so to speak, for context comparison computations at Level 3.

(b) Different local circuits may code for different aspects of the expected or current context.

(a) Local circuit information provides input to this larger population computation that determines the degree of similarity between what is expected to occur in a given context, and what is actually happening.

(b) Hippocampal output impacts neocortical memory and behavioral expression systems, which in turn impacts subsequent $\mathrm{M}$ input to place cells.

While Level 1 is considered a discrete input stage for the hippocampal analysis of context, Levels 2 and 3 may be graded such that there are from very precise to more broad information evaluated by different local and population circuits. Thus, there are likely multiple Level 2 and 3 circuits distributed across hippocampus.

behavior and neural plasticity mechanisms (e.g. Buzsáki, 2005; Zugaro et al., 2005).

Another form of temporal-based neuroplasticity within hippocampus involves a change in the timing of spike discharge by one cell relative to spiking of other cells, or relative to prior spike discharges by the same cell. For example, it has been shown that the temporal coherence of place cell discharge is greater in mice with an intact hippocampus compared to mice with deficient NMDA systems (McHugh et al., 1996). Greater synchronization could offer a stronger output signal to efferent structures. Relatedly, experience-dependent temporal codes may be found in terms of the temporal relationships between the firing of cells with adjacent place fields. With continued exposure to a new environment, place fields begin to expand asymmetrically such that the peak firing rate occurs with shorter latency upon entrance into the field (Mehta et al., 1997, 2000). The asymmetric backward expansion of place fields is thought to provide a neural mechanism for learning directional sequences. Moreover, it has been suggested that the backward expansion phenomenon may contribute to the transformation of a rate code to a temporal code such as that illustrated in phase precession (Mehta et al., 2002). Perhaps, the backward expansion phenomenon could help to explain other place field phenomenon such as the tendency for place cells to fire in anticipation of entering a specified location within a familiar environment (Muller and Kubie, 1989).

While the dynamic changes in place field shape are intriguing, it remains a challenge to determine whether, and then how, changes in the temporal distribution of cell firing are directly related to spatial learning, and how these alterations are reflected in local circuit codes (Level 2). However, Shapiro and Ferbinteanu (2006) recently reported that the temporal relationship between firing of simultaneously recorded place cells discriminated task phase, suggesting that past experience determines relative spike timing of individual neurons. Experience-dependent changes in spike timing are evident not only when considering cell discharge of one cell relative to another, or to itself, but also timing relative to task-specific events. Lee et al. (2006) described place fields whose center of mass moved in the forward direction (toward a goal) with each successive trial. The direction of place field movement was in the direction opposite to that predicted by spike timing dependent plasticity mechanisms. However, it may be that place fields will move in the forward or backward directions for different purposes, and their relative direction of movement may depend on the task conditions. For example, forward movement of place fields may be more prevalent when choices must be made between multiple goal locations. Backward movement may be more prominent when merely knowing the direction of movement is sufficient to predict future reinforcement. In these cases, a distinguishing feature would not only be the direction of place field movement, but also the temporal resolution of the movement itself. Indeed, forward movement of place fields seemed to occur over a protracted time scale relative to the backward expansion effect. If the above analysis is correct, then a critical issue is to determine the mechanism that regulates place field shifts in the forward and backward directions, and whether these are independent mechanisms. It is also possible that the more protracted forward movement of place fields reflects changes in the spatial code instead of, or in addition to, a temporal shift.

Ferbinteanu and Shapiro (2003) described place fields that were consistently located on the goal arm of a plus maze regardless of the start location. These cells were considered to represent prospective information about the upcoming reward event. An 
earlier example of prospective coding by place cells was the demonstration that place fields seemed to anticipate the future location of an animal by some $90 \mathrm{~ms}$ (Muller and Kubie, 1989). The time scales for the latter two studies are very different so it remains to be determined if they are derived from the same or different mechanism. One may also consider the backward expansion effect (described above) a form of anticipatory neural code due to $\mathrm{M}$ input. It is possible, then, that at least a subpopulation of place cells conveys information about what to expect in the future within the milliseconds to seconds range. Place cells may also code information retrospectively. Place fields have been found at consistent distances from a start location on linear tracks and plus mazes (e.g. Gothard et al., 1996; Ferbinteanu and Shapiro, 2003). In summary, these findings are consistent with the view that changes in spike timing may allow hippocampal cells to encode perhaps simultaneously information about what to expect in a given context (prospective code), as well as current or recent (retrospective) events.

CONTEXT DISCRIMINATION BY HIPPOCAMPUS IS GUIDED BY, AND ALSO INFORMS, A BROADER CONTEXT PROCESSING SYSTEM

Information about the stability or changes in context is likely to be useful to insure adaptive behavioral response selection and execution, and to update currently active memory or other intrinsic processing systems. Thus, it should be expected that hippocampal context discrimination functions operate continuously and automatically almost regardless of the task at hand. This is not to say, however, that task demands don't regulate the efficiency or perhaps even the type of hippocampal context information being compared. In fact, the contrary may be true.

\section{Entorhinal Cortex-Hippocampal Interactions}

The preponderance of striking place fields regardless of the cognitive strategy required by navigating rats (e.g. Eschenko and Mizumori, 2007) suggests that hippocampus is somehow predisposed to organize contextual information spatially. Place field experiments invariably involve exploration of the environment. Such voluntary movement may direct spatial and movement signals through the medial entorhinal cortex such that networks of spatial grid cells become activated (Hafting et al., 2005; Leutgeb S et al., 2005; Sargolini et al., 2006). Grid cells selectively discharge when rats traverse positions that coincide with the vertices of a regularly tessellating grid. The grid fields appear to represent conjunctions of location, direction, and movement information within a twodimensional coordinate representation of the environment. Thus, the entorhinal cortex may pass on to hippocampus a spatial framework (McNaughton et al., 2006) within which context-specific M and $\mathrm{T}$ information is organized and processed. For this reason, the term spatial context is often preferred when discussing context processing during navigation (Nadel et al., 1985; Mizumori et al., 1999b, 2000; Jeffery et al., 2004).
The notion of exploration-induced spatial organization of hippocampal representations is consistent with the finding that hippocampal place fields are observed upon first exposure to a new environment (Muller and Kubie, 1987; Wilson and McNaughton, 1993; Markus et al., 1995; O’Keefe and Burgess, 1996a,b; Hetherington and Shapiro, 1997; Frank et al., 2004). Also, compared to passive movement conditions in which rats are made to go through a place field either by being held by the experimenter or by being placed on a moveable robotic device, active and unrestrained movement seems to generate more selective and reliable place fields (Foster et al., 1989; Gavrilov et al., 1998; Song et al., 2005). Furthermore, pyramidal cells fire more robustly when rats run faster across a given location (i.e. running in a running wheel; Czurko et al., 1999). Voluntary locomotion, and the subsequent imposition of a spatial organization to contextual information, appears to have the effect of sharpening the neural image of spatial representations, at least in dorsal hippocampus. A sharper code should relay more specific spatial information to intrinsic and extrinsic computational circuits. The finding that unrestrained movement produced sharper codes than passive movement suggests that learned behavioral responses have more meaning for, and impact on, place fields than random movement of the animal. This may be one way in which learned information helps to define a context code.

The tendency for voluntary navigation to impose a spatial organization of contextual information may also explain why place fields have not been seen as the predominant form of coding in the primate hippocampus. Monkey hippocampal neurons respond primarily when the subject directs its gaze at a particular part of the environment (Rolls, 1999), whereas rodents explore the environment by active locomotion, primates accomplish much of their exploration visually, by directing their gaze about the environment. Using virtual navigation methods, location-selective firing has not only been reported, but it seems selective to contexts in which subjects must search for and identify meaningful locations (Ekstrom et al., 2003). Thus, the apparently discrepant findings between primate, human, and rodent studies may not indicate fundamentally different computations by the hippocampus, but rather they may result from different combinations of information passed on to hippocampus, information that is dictated by the task conditions.

Interestingly, a common feature of the neural organization of most sensory systems is that there is a clear spatial organization (or topography) to neural representations, starting from the sensory receptor to sensory cortex. This form of organization may facilitate adaptive responding since motor output systems (from motor cortical areas through spinal cord) are also organized topographically. Assuming that the use of a spatial reference frame to organize sensory and response information is highly efficient, it seems reasonable to speculate that there was strong evolutionary pressure to process and retain sensory information spatially as sensory association (cortical) regions evolved. Maintaining a spatial reference framework for fundamentally important tasks such as accurate navigation may have facilitated an organism's ability to rapidly adapt to environmental changes. For this reason, phylogenetically old structures such as hippocampus may be initially predisposed to process information within a spatial framework, regardless of the 
task. Indeed, hippocampal place fields are observed with similar abundance in spatial and nonspatial tasks (Yeshenko et al., 2004). The fact that it is possible to break out of the spatial framework if needed may reflect a more recently evolved adaptation.

Temporal organization of spatially organized hippocampal neural codes appears to be reflected in neural signaling by neocortical cells. It was shown that the temporal sequence of neural firing recorded during exploration is essentially "replayed" during slow wave sleep but in a temporally condensed fashion (Wilson and McNaughton, 1994; Hoffman and McNaughton, 2002; Pennartz et al., 2002). Recently, another form of replay of neural activation was reported when rats paused between traversals along an elevated track (Foster and Wilson, 2006). This differed from the replay observed during sleep because the temporal order of neural activation was reversed relative to the order in which locations were visited during locomotion. This reverse replay was interpreted as being useful for a different mnemonic function, one that allows the evaluation of recent behaviors (trajectories in this case) in terms of their reinforcement outcomes. This particular form of temporal organization may be generated within hippocampus, reflecting a retrospective mechanism that is needed to detect changes in the expected situation.

The different forms of temporal coordination can be used to predict behaviors and events in a prospective manner, or to recall recent behaviors or events in a retrospective manner. Thus, $\mathrm{T}$ information can be represented in the multidimensional context matrix (Fig. 2) according to the degree to which the neural code is prospective or retrospective. Cell-to-cell spike timing changes likely emerge from a number of natural synaptic sources that regulate coordinated spike activity within hippocampus. More broadly speaking, rhythmic activity may be regulated by plastic changes within intrahippocampal circuitry and by extrinsic systems responsible for generating rhythmic activity in hippocampus (e.g. brainstem: Buzsáki, 2002, 2005; Vertes et al., 2004; Hasselmo, 2005). The varied sources of temporal regulation may allow for coding such $\mathrm{M}$ information as stimulus sequences (Dragoi and Buzsáki, 2006), or for determining stimulus duration.

\section{Retrosplenial (Parietal) Cortex-Hippocampal Interactions}

The parietal cortex is traditionally considered in terms of space-based attention to the external environment as well as attention to oneself (Colby and Goldberg, 1999). Movement intention is another commonly considered function of parietal cortex. Recent findings, however, indicate that at least the retrosplenial sector of parietal cortex may serve more complex cognitive roles. For example, retrosplenial cortex is one of the many posterior parietal structures that become active during correct responses in item recognition tasks (Konishi et al., 2000), suggesting a critical role in memory retrieval (Wagner et al., 2005). In the case of spatial navigation by rats, the retrosplenial cortex has been discussed as a key player in updating visuospatial representations with movement-related (idiothetic) information (Sutherland et al., 1988; Cooper and Mizumori, 2001; Harker and Whishaw, 2004). Anatomical connections of the retrosplenial cortex support such an integrative role between visual and

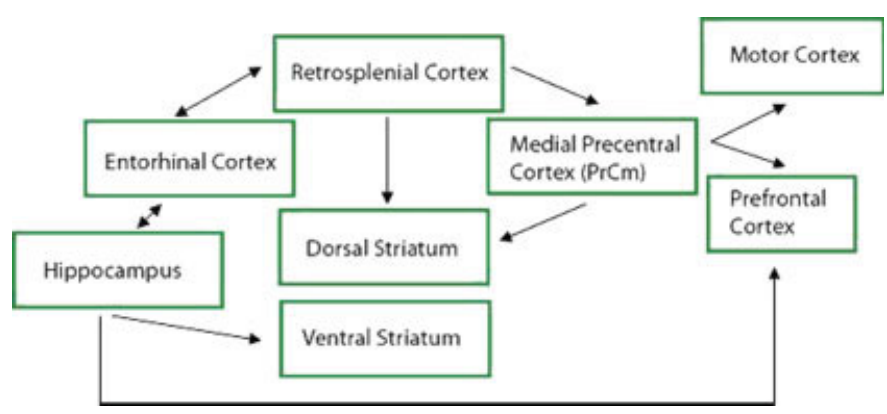

FIGURE 5. Schematic illustration of the neural circuitry that provides hippocampus information needed to establish context representations. Also shown are neocortical and striatal circuitries that likely receive hippocampal output messages to affect ongoing behavioral and cognitive strategies (details are provided in text). [Color figure can be viewed in the online issue, which is available at www.interscience.wiley.com.]

movement-related information since it receives input from, and returns projections to, visual association cortex, medial precentral cortex, motor cortex, anterior cingulate cortex, and the subicular complex (Vogt and Miller, 1983; Wyss and van Groen, 1992; see Fig. 5). Temporary inactivation of the retrosplenial cortex impairs spatial performance on a radial maze when darkness was imposed on a familiar test environment, but not when the same environment was lit (Cooper and Mizumori, 2001). The same inactivation procedure impaired initial learning in a well lit room. Thus, it was concluded that the retrosplenial cortex contributes to spatial navigation when learned associations between visual and idiothetic information are required to perform the task (as in the case of well trained rats performing without familiar visual cues), or when new associations are being formed between visual and idiothetic information (as in the case of new learning in a lit environment). That retrosplenial information importantly informs hippocampal processing was shown by the finding that retrosplenial cortex inactivation altered hippocampal place fields that were recorded during the same experiment. It appears then that retrosplenial cortex may serve as perhaps one of many portals through which hippocampal information interacts with stored memory representations: Retrosplenial cortex may relay to hippocampus context-specific integrated sensory and movement information. Hippocampus in turn may effectively update the memory representations by providing retrosplenial cortex with feedback about the reliability of the context-based input.

To test the view that retrosplenial cortex provides integrated learned context information to hippocampus in the service of context discrimination, Smith et al. (2003) recorded retrosplenial cortex neurons as rats learned to discriminate behavioral contexts that differed only in terms of the location of food reward. That is, sensory and behavioral aspects were the same for the two contexts. It was found that reward-related retrosplenial cortex neurons developed task-relevant responses when animals learned the context discrimination (see Fig. 6 for examples), but not when rats foraged for randomly located food on the same maze. This result is entirely consistent with Smith et al. (2004) who showed 

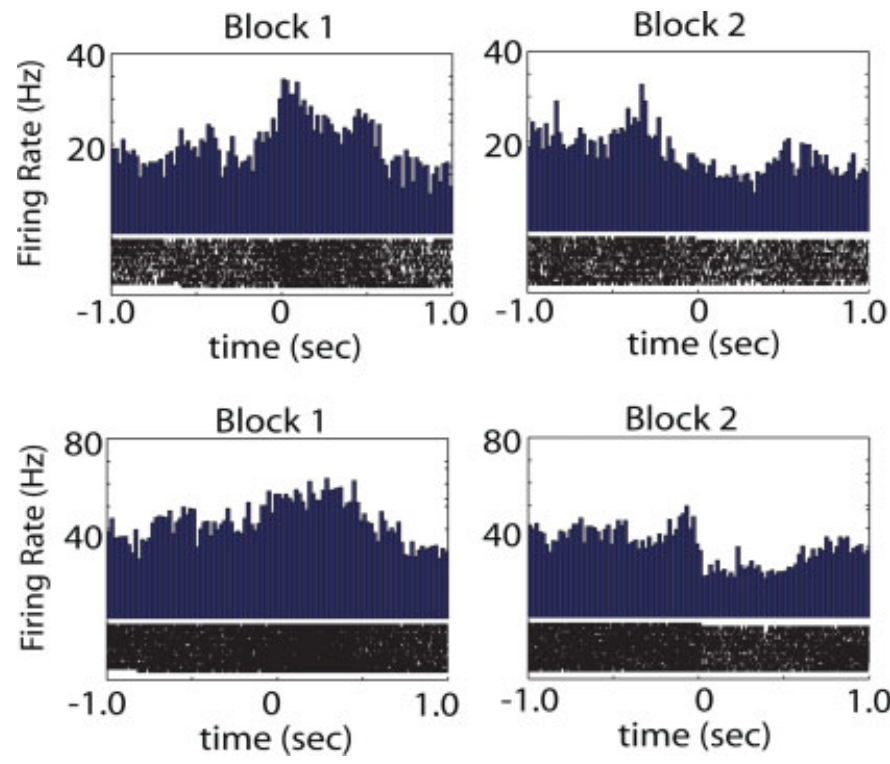

FIGURE 6. Event histograms illustrating context-specific responding by reward sensitive retrosplenial cortex neurons. Details of the training can be found in the text. Briefly, rats were trained to discriminate contexts according to where (on a plus maze) the animal expected to find reward. Top row: This cell showed elevated firing associated with reward consumption during trials 1-10 trials in one context (Block 1), while showing inhibited responding during trials 11-20 performed in the second context (Block 2). All trials occurred during the same recording session and within the same sensory environment. Bottom row: This cell showed only a slight elevation in firing during reward consumption during Block 1, while showing a marked reduction in firing rate during Block 2 . These data demonstrate that, like hippocampus (Smith and Mizumori, 2006b), retrosplenial cortex neurons discriminate learned contexts. [Color figure can be viewed in the online issue, which is available at www.interscience.wiley.com.]

that disconnection of retrosplenial cortex and hippocampus resulted in a loss of context-specific firing by retrosplenial cortex neurons. This pattern of neural responses, together with similar responses reported for hippocampal neurons (Smith and Mizumori, 2006b), indicates that a context discrimination circuit extends beyond hippocampus. Moreover, hippocampal retrieval of context-specific knowledge and behaviors may be mediated by retrosplenial cortex.

Consistent with previous suggestions (e.g. Sutherland and Hoesing, 1993; McNaughton et al., 1996) that posterior neocortical regions contribute to the determination of the appropriate cognitive and behavioral strategies to be used by the animal, retrosplenial cortex may serve another key function relevant to context discrimination, and that is to facilitate the appropriate behavioral responses when changes in context are detected. From retrosplenial cortex, updated information may be passed on to striatum (Risold and Swanson, 1995), or to premotor areas of cortex (Reep et al., 1990). An important relay in the latter pathway may include the medial precentral nucleus, or $\operatorname{PrCm}$ (also referred to as medial agranular cortex, Reep et al., 1987; Reep and Corwin, 1999). Indeed, we have shown that reversible inactivation of the retrosplenial cortex produces significant alteration of $\mathrm{PrCm}$ neural correlates (Mizumori et al., 2002; Mizumori et al., under review).
The $\mathrm{PrCm}$ may play a pivotal role in the implementation of future cognitive strategies (Mizumori et al., 2002; Mizumori et al., under review) for it projects to the striatum (Reep et al., 1987; Reep and Corwin, 1999) as well as to frontal cortical motor regions (Reep et al., 1990). The PrCm-striatal projection could provide spatial context-dependent movement information to the striatum. The striatum may in turn evaluate the reinforcement consequences of the current behavior that is engaged within a defined context (e.g. Mizumori et al., 1999a; Schultz and Dickinson, 2000). It would be highly adaptive for this striatal analysis to continue through new learning as well as during asymptotic performance. This would allow continual feedback from striatum to cortex regarding the effectiveness of behavioral/ cognitive strategies. If the $\mathrm{PrCm}$ signal is one that indicates that the context has changed, striatum may become primed for maximal sensitivity to impending input. Such feedback seems critical for an animal's ability to quickly respond to changes in a familiar environment. For situations in which the strategy remains constant (i.e. during asymptotic performance in a static environment), behaviors can be implemented more quickly via direct $\mathrm{PrCm}$ efferent connection to motor cortex. The PrCm, then, may allow organisms to quickly respond to changes in familiar situations as well as to "automate" their responses in welllearned, stable situations.

Connections between PrCm and the prefrontal cortex (PFC) provide what might be considered as the last link in a polysynaptic series of connections between hippocampus and cortical circuitry that is thought to be essential for implementing and orchestrating adaptive behaviors. With this indirect pathway, memory guided interpretations of changes in the expected context (via the indirect path) can be integrated with direct signals from hippocampus (via direct hippocampal-PFC connections; Jay and Witter, 1991) that signal occurence of a change. Perhaps the latter serves to transform PFC neurons into an "up state" of cell excitability so that it can readily incorporate subsequent information coming in from the indirect pathway. Consistent with this view, PFC neurons exhibit context-dependent anticipatory neural codes for reward information (Pratt and Mizumori, 2001). Also, PFC neural signaling has been found to be conditionally linked to hippocampal output since PFC cell firing can become phase locked to CA1 hippocampal theta rhythms and precession (Hyman et al., 2005; Jones and Wilson, 2005). The entrained PFC neurons tended to be ones whose firing is correlated with specific behavioral acts (Hyman et al., 2005). Furthermore, Hyman et al. showed that testing subjects in different rooms caused some PFC neurons to change the degree to which they were entrained to the hippocampal theta rhythm, suggesting that the degree to which PFC is functionally connected to HPC was dependent on the stability of spatial context information

\section{CONCLUSION}

Although it is abundantly clear that the hippocampus engages in context processing, it is less certain how and to what extent this context analysis contributes to the broader cortical learning cir- 
cuit. Hippocampus may serve to evaluate the extent to which one's expectations about contextual features of a situation are met. In navigating animals, such an evaluation requires not only an input from neocortical memory systems to define the expected context, but such information is subject to spatial and temporal organization via temporal (entorhinal) cortical and subcortical input. The outcome of hippocampal analyses is used to update subsequent memory activation networks in cortex and to guide ongoing behavioral acts. Both of these functions may involve the retrosplenial cortex. Recent evidence suggests that retrosplenial cortex is especially related to accurate behavioral choice when current visuomotor input must be linked to past visuomotor associations. Thus, the retrosplenial cortex may serve as one of perhaps many portals through which memory representations can be accessed.

To affect behavioral choice, it is suggested that the direct pathway from hippocampus to PFC serves to change frontal cortex to an excitable state that is ready to quickly respond to the new information arriving via the indirect pathway that includes retrosplenial cortex and the medial precentral nucleus ( $\mathrm{PrCm})$. Together, the $\mathrm{PrCm}$ and the PFC coordinate a striatal-frontal cortical information system that evaluates the reinforcement consequences of the new context, and then alters the behavioral or cognitive strategy if needed. Many aspects the present proposal are generally in line with different existing theories of hippocampal, entorhinal, retrosplenial or frontal cortical function that are based largely on lesion studies. The electrophysiological approach has generated a large body of evidence that converges onto the view that hippocampus serves to discriminate spatial contexts. The significance of such context discrimination is becoming better appreciated as neural recordings expand to include neocortical striatal regions. In this way, the spatial navigation model of learning and memory continues to provide new insight regarding basic principles of neuron and neural system functional organization.

\section{Acknowledgments}

Relevant to the topic of this article, we gratefully acknowledge many thoughtful discussions by numerous outstanding students. The most recent students include Oxana Eschenko, Adria Martig, Katy Gill, and Min Jung Kim.

\section{REFERENCES}

Anagnostaras SG, Gale GD, Fanselow MS. 2001. Hippocampus and contextual fear conditioning: Recent controversies and advances. Hippocampus 11:8-17.

Anderson MI, Jeffery KJ. 2003. Heterogeneous modulation of place cell firing by changes in context. J Neurosci 23:8827-8835.

Bouton ME, Westbrook RF, Corcoran KA, Maren S. 2006. Contextual and temporal modulation of extinction: Behavioral and biological mechanisms. Biol Psychia 60:352-360.

Burgess N, Becker S, King JA, O'Keefe J. 2001. Memory for events and their spatial context: Models and experiments. Philos Trans R Soc Lond B Biol Sci 356:1493-1503.

Buzsáki G. 2002. Theta oscillations in the hippocampus. Neuron 33:325-340.
Buzsáki G. 2005. Theta rhythm of navigation: Link between path integration and landmark navigation, episodic and semantic memory. Hippocampus 15:827-840.

Colby CL, Goldberg ME. 1999. Space and attention in parietal cortex. Ann Rev Neurosci 22:319-349.

Cooper BG, Mizumori SJY. 2001. Temporary inactivation of retrosplenial cortex causes a transient reorganization of spatial coding in hippocampus. J Neurosci 21:3986-4001.

Czurkó A, Hirase H, Csicsvari J, Buzsáki G. 1999. Sustained activation of hippocampal pyramidal cells by 'space clamping' in a running wheel. Eur J Neurosci 11:344-352.

Dragoi G, Buzsáki G. 2006. Temporal encoding of place sequences by hippocampal cell assemblies. Neuron 50:145-157.

Eichenbaum H, Cohen NJ. 2001. From Conditioning to Conscious Recollection: Memory Systems of the Brain. New York: Oxford University Press.

Eichenbaum H, Dudchenko P, Wood E, Shapiro M, Tanila H. 1999. The hippocampus, memory, and place cells: Is it spatial memory or a memory space? Neuron 425:184-188.

Ekstrom AD, Kahana MJ, Caplan JB, Fields TA, Isham EA, Newman EL, Fried I. 2003. Cellular networks underlying human spatial navigation. Nature 425:184-188.

Eschenko O, Mizumori SJY. 2007. Memory influences on hippocampal and striatal neural codes: Effects of a shift between task rules. Neurobiol Learn Mem 495-509.

Fanselow MS, Poulos AM. 2005. The neuroscience of mammalian associative learning. Ann Rev Psychol 56:207-234.

Ferbinteanu J, Shapiro ML. 2003. Prospective and retrospective memory coding in the hippocampus. Neuron 40:1227-1239.

Fortin NJ, Agster KL, Eichenbaum HB. 2002. Critical role of the hippocampus in memory for sequences of events. Nat Neurosci 5:458-462.

Foster DJ, Wilson MA. 2006. Reverse replay of behavioural sequences in hippocampal place cells during the awake state. Nature 440:680-683.

Foster TC, Christian EP, Hampson RE, Campbell KA, Deadwyler SA. 1987. Sequential dependencies regulate sensory evoked responses of single units in the rat hippocampus. Brain Res 408:86-96.

Foster TC, Castro CA, McNaughton BL. 1989. Spatial selectivity of rat hippocampal neurons: Dependence on preparedness for movement. Science 244:1580-1582.

Frank LM, Stanley GB, Brown EN. 2004. Hippocampal plasticity across multiple days of exposure to novel environments. J Neurosci 24:7681-7689.

Freeman JH Jr, Cuppernell C, Flannery K, Gabriel M. 1996a. Contextspecific multi-site cingulate cortical, limbic thalamic, and hippocampal neuronal activity during concurrent discriminative approach and avoidance training in rabbits. J Neurosci 16:1538-1549.

Freeman JH Jr, Cuppernell C, Flannery K, Gabriel M. 1996b. Limbic thalamic, cingulate cortical and hippocampal neuronal correlates of discriminative approach learning in rabbits. Behav Brain Res 80:123-136.

Fyhn M, Molden S, Hollup S, Moser MB, Moser E. 2002. Hippocampal neurons responding to first-time dislocation of a target object. Neuron 35:555-566.

Gavrilov VV, Wiener SI, Berthoz A. 1998. Discharge correlates of hippocampal complex spike neurons in behaving rats passively displaced on a mobile robot. Hippocampus 8:475-490.

Good M, Honey RC. 1991. Conditioning and contextual retrieval in hippocampal rats. Behav Neurosci 105:499-509.

Gothard KM, Skaggs WE, Moore KM, McNaughton BL. 1996. Binding of hippocampal CA1 neural activity to multiple reference frames in a landmark-based navigation task. J Neurosci 16:825-835.

Gray JA. 1982. The Neuropsychology of Anxiety: An Enquiry into the Functions of the Septo-hippocamal System. Oxford: Oxford University Press.

Gray JA. 2000. The Neuropsychology of Anxiety: An Enquiry into the Functions of the Septo-hippocamal System, 2nd ed. Oxford: Oxford University Press. 
Hafting T, Fyhn M, Molden S, Moser MB, Moser EI. 2005. Microstructure of a spatial map in the entorhinal cortex. Nature 436:801-806.

Harker KT, Whishaw IQ. 2004. A reaffirmation of the retrosplenial contribution to rodent navigation: Reviewing the influences of lesion, strain, and task. Neurosci Biobehav Rev 28:485-496.

Hasselmo ME. 2005. What is the function of hippocampal theta rhythm?-Linking behavioral data to phasic properties of field potential and unit recording data. Hippocampus 15:936-949.

Hasselmo ME, Hay J, Ilyn M, Gorchetchnikov A. 2002. Neuromodulation, theta rhythm and rat spatial navigation. Neural Netw 15:689-707.

Hetherington PA, Shapiro ML. 1997. Hippocampal place fields are altered by the removal of single visual cues in a distance-dependent manner. Behav Neurosci 11:20-34.

Hirsh R. 1974. The hippocampus and contextual retrieval of information from memory: A theory. Behav Biol 12:421-444.

Hoffman KL, McNaughton BL. 2002. Sleep on it: Cortical reorganization after-the-fact. Trends Neurosci 25:1-2.

Hyman JM, Zilli EA, Paley AM, Hasselmo ME. 2005. Medial prefrontal cortex cells show dynamic modulation with the hippocampal theta rhythm dependent on behavior. Hippocampus 15: 739-749.

Jay TM, Witter MP. 1991. Distribution of hippocampal CA1 and subicular efferents in the prefrontal cortex of the rat studied by means of anterograde transport of Phaseolus vulgaris-leucoagglutinin. J Comp Neurol 313:574-586.

Jeffery KJ, Anderson MI, Hayman R, Chakraborty S. 2004. A proposed architecture for the neural representation of spatial context. Neurosci Biobehav Rev 28:201-218.

Jenkins TA, Amin E, Pearce JM, Brown MW, Aggleton JP. 2004. Novel spatial arrangements of familiar visual stimuli promote activity in the rat hippocampal formation but not the parahippocampal cortices: A c-fos expression study. Neuroscience 124:43-52.

Jones MW, Wilson MA. 2005. Theta rhythms coordinate hippocampal-prefrontal interactions in a spatial memory task. PLoS Biol 3:e402.

Kim JJ, Fanselow MS. 1992. Modality-specific retrograde amnesia of fear. Science 256:675-677.

Konishi S, Wheeler ME, Donaldson DI, Buckner RL. 2000. Neural correlates of episodic retrieval success. Neuroimage 12:276-286.

Lee I, Griffin AL, Zilli EA, Eichenbaum H, Hasselmo ME. 2006. Gradual translocation of spatial correlates of neuronal firing in the hippocampus toward prospective reward locations. Neuron 51:639-650.

Leutgeb JK, Leutgeb S, Treves A, Meyer R, Barnes CA, McNaughton BL, Moser MB, Moser EI. 2005. Progressive transformation of hippocampal neuronal representations in "morphed" environments. Neuron 48:345-358.

Leutgeb S, Leutgeb JK, Barnes CA, Moser EI, McNaughton BL, Moser MB. 2005. Independent codes for spatial and episodic memory in hippocampal neuronal ensembles. Science 309:619-623.

Lisman JE, Otmakhova NA. 2001. Storage, recall, and novelty detection of sequences by the hippocampus: Elaborating on the SOCRATIC model to account for normal and aberrant effects of dopamine. Hippocampus 11:551-568.

Manns JR, Zilli EA, Ong KC, Hasselmo ME, Eichenbaum H. 2007. Hippocampal CA1 spiking during encoding and retrieval: Relation to theta phase. Neurobiol Learn Mem 87:9-20.

Maren S. 2001. Neurobiology of Pavlovian fear conditioning. Ann Rev Neurosci 2:897-931.

Markus EJ, Qin YL, Leonard B, Skaggs WE, McNaughton BL, Barnes CA. 1995. Interactions between location and task affect the spatial and directional firing of hippocampal neurons. J Neurosci 15: 7079-7094.

McHugh TJ, Blum KI, Tsien JZ, Tonegawa S, Wilson MA. 1996. Impaired hippocampal representation of space in CA1-specific NMDAR1 knockout mice. Cell 87:1339-1349.
McNaughton BL, Barnes CA, O’Keefe J. 1983. The contributions of position, direction, and velocity to single unit activity in the hippocampus of freely-moving rats. Exp Brain Res 52:41-49.

McNaughton BL, Barnes CA, Gerrard JL, Gothard K, Jung MW, Knierim JJ, Kudrimoti H, Qin Y, Skaggs WE, Suster M, Weaver KL. 1996. Deciphering the hippocampal polyglot: The hippocampus as a path integration system. J Exp Biol 199:173-185.

McNaughton BL, Battaglia FP, Jensen O, Moser EI, Moser MB. 2006. Path integration and the neural basis of the 'cognitive map'. Nat Rev Neurosci 7:663-78

Mehta MR, Barnes CA, McNaughton BL. 1997. Experience-dependent, asymmetric expansion of hippocampal place fields. Proc Natl Acad Sci USA 94:8918-8921.

Mehta MR, Quirk MC, Wilson MA. 2000. Experience-dependent asymmetric shape of hippocampal receptive fields. Neuron 25:707-715.

Mehta MR, Lee AK, Wilson MA. 2002. Role of experience and oscillations in transforming a rate code into a temporal code. Nature 417:741-746.

Mizumori SJY. 2007. A context for hippocampal place cells during learning. In: Mizumori SJY, editor. Hippocampal Place Cells: Relevance to Learning and Memory. Oxford University Press. New York, NY.

Mizumori SJY, Kalyani A. 1997. Age and experience-dependent representational reorganization during spatial learning. Neurobiol Aging 18:651-659.

Mizumori SJY, Pratt WE, Ragozzino KE. 1999a. Function of the nucleus accumbens within the context of the larger striatal system. Psychobiology 27:214-224.

Mizumori SJY, Ragozzino KE, Cooper BG, Leutgeb S. 1999b. Hippocampal representational organization and spatial context. Hippocampus 9:444-451.

Mizumori SJY, Cooper BG, Leutgeb S, Pratt WE. 2000. A neural systems analysis of adaptive navigation. Mol Neurobiol 21:57-82.

Mizumori SJY, Pratt WE, Cooper BG, Guazzelli A. 2002. The behavioral implementation of hippocampal processing. In: Sharp PE, editor. The Neural Basis of Navigation: Evidence from Single Cell Recording. Boston, MA: Kluwer Academic. pp 197-216.

Mizumori SJY, Smith DM, Puryear CB. 2007. Mnemonic contributions of hippocampal place cells. In: Martinez JL, Kesner RP, editors. Neurobiology of Learning and Memory. Burlington, MA: Academic, pp 155-189.

Moita MA, Rosis S, Zhou Y, LeDoux JE, Blair HT. 2004. Putting fear in its place: Remapping of hippocampal place cells during fear conditioning. J Neurosci 24:7015-7023.

Morris RGM. 2001. Episodic-like memory in animals psychological criteria, neural mechanisms and the value of episodic-like tasks to investigate animal models of neurodegenerative disease. Philos Trans R Soc Lond B Biol Sci 356:1453-1465.

Muller RU, Kubie JL. 1987. The effects of changes in the environment on the spatial firing of hippocampal complex-spike cells. J Neurosci 7:1951-1968.

Muller RU, Kubie JL. 1989. The firing of hippocampal place cells predicts the future position of freely moving rats. J Neurosci 9:4101-4110.

Myers CE, Gluck M. 1994. Context, conditioning, and hippocampal representation in animal learning. Behav Neurosci 108:835-847.

Nadel L, Payne JD. 2002. The hippocampus, wayfinding and episodic memory. In: Sharp PE, editor. The Neural Basis of Navigation: Evidence from Single Cell Recording. Boston, MA: Kluwer Academic. pp 235-248.

Nadel L, Wilner J. 1980. Context and conditioning: a place for space. Physiol Psychol 8:218-228.

Nadel L, Willner J, Kurz EM. 1985. Cognitive maps and environmental context. In: Balsam P, Tomie A, editors. Context and Learning. Hillside, NJ: Erlbaum. pp 385-406.

O'Keefe J. 1976. Place units in the HPC of the freely moving rat. Exp Neurol 51:78-109. 
O'Keefe J, Burgess N. 1996a. Geometric determinants of the place fields of hippocampal neurons. Nature 381:425-428.

O'Keefe J, Burgess N. 1996b. Spatial and temporal determinants of hippocampal place cell activity. In: Ono T, McNaughton BL, Molotchnikoff S, Rolls ET, Nishijo H, editors. Perception, Memory and Emotion: Frontiers in Neuroscience. New York: Pergamon Press. pp 359-373.

O'Keefe J, Nadel L. 1978. The Hippocampus as a Cognitive Map. Oxford: Oxford University Press.

O’Keefe J, Recce ML. 1993. Phase relationship between hippocampal place units and the EEG theta rhythm. Hippocampus 3:317-330.

O'Reilly RC, Rudy JW. 2001. Conjunctive representations in learning and memory: Principles of cortical and hippocampal function. Psychol Rev 108:311-345.

Paulsen O, Moser EI. 1998. A model of hippocampal memory encoding and retrieval: GABAergic control of synaptic plasticity. Trends Neurosci 21:273-278.

Penick S, Solomon PR. 1991. Hippocampus, context, and conditioning. Behav Neurosci 105:611-617.

Pennartz CM, Uylings HB, Barnes CA, McNaughton BL. 2002. Memory reactivation and consolidation during sleep: From cellular mechanisms to human performance. Prog Brain Res 138:143166.

Phillips RG, LeDoux JE. 1992. Differential contribution of amygdala and hippocampus to cued and contextual fear conditioning. Behav Neurosci 106:274-285.

Phillips RG, LeDoux JE. 1994. Lesions of the dorsal hippocampal formation interfere with background but not foreground contextual fear conditioning. Learn Mem 1:34-44.

Pratt WE, Mizumori SJY. 2001. Neurons in rat medial prefrontal cortex show anticipatory rate changes to predictable differential rewards in a spatial memory task. Behav Brain Res 123:165-183.

Puryear CB, King M, Mizumori SJY. 2006. Specific changes in hippocampal spatial codes predict spatial working memory performance. Behav Brain Res 169:168-175.

Redish AD, Rosesnzweig ES, Bohanick JD, McNaughton BL, Barnes CA. 2000. Dynamics of hippocampal ensemble activity realignment: Time versus space. J Neurosci 20:9298-9309.

Reep RL, Corwin JV. 1999. Topographic organization of the striatal and thalamic connections of rat medial agranular cortex. Brain Res 841:43-52.

Reep RL, Corwin JV, Hashimoto A, Watson RT. 1987. Efferent connections of the rostral portion of medial agranular cortex in rats. Brain Res Bull 19:203-221.

Reep RL, Goodwin GS, Corwin JV. 1990. Topographical organization in the corticocortical connections of medial agranular cortex in rats. J Comp Neurol 294:262-280.

Risold PY, Swanson LW. 1995. Evidence for a hypothalamothalamocortical circuit mediating pheromonal influences on eye and head movements. Proc Nat Acad Sci USA 92:3898-3902.

Rolls ET. 1999. Spatial view cells and the representation of place in the primate hippocampus. Hippocampus 9:467-480.

Sargolini F, Fyhn M, Hafting T, McNaughton BL, Witter MP, Moser MB, Moser EI. (2006). Conjunctive representation of position, direction, and velocity in entorhinal cortex. Science 312:758-762.

Save E, Buhot MC, Foreman N, Thinus-Blanc C. 1992a. Exploratory activity and response to a spatial change in rats with hippocampal or posterior parietal cortical lesions. Behav Brain Res 47:113-127

Save E, Poucet B, Foreman N, Buhot MC. 1992b. Object exploration and reactions to spatial and nonspatial changes in hooded rats following damage to parietal cortex or hippocampal formation. Behav Neurosci 106:447-456.

Schultz W, Dickinson A. 2000. Neuronal coding of prediction errors. Ann Rev Neurosci 23:473-500.

Shapiro ML, Eichenbaum H. 1999. Hippocampus as a memory map: Synaptic plasticity and memory encoding by hippocampal neurons. Hippocampus 9:365-384.
Shapiro ML, Ferbinteanu J. 2006. Relative spike timing in pairs of hippocampal neurons distinguishes the beginning and end of journeys. Proc Natl Acad Sci USA 103:4287-4292.

Shapiro ML, Tanila H, Eichenbaum H. 1997. Cues that hippocampal place cells encode: Dynamic and hierarchical representation of local and distal stimuli. Hippocampus 7:624-642.

Shors TJ, Matzel LD. 1997. Long-term potentiation: What's learning got to do with it? Behav Brain Sci 20:597-614.

Skaggs WE, McNaughton BL, Wilson MA, Barnes CA. 1996. Theta phase precession in hippocampal neuronal populations and the compression of temporal sequences. Hippocampus 6:149-172.

Smith DM, Mizumori SJY. 2006a. Hippocampal place cells, context, and episodic memory. Hippocampus 16:716-729.

Smith DM, Mizumori SJY. 2006b. Learning-related development of context-specific neuronal responses to places and events: The hippocampal role in context processing. J Neurosci 26:3154-3163.

Smith DM, Munoz J, Turner T, Mizumori SJY. 2003. Spatial, directional, reward, and context-related responses of retrosplenial cortical and anterior thalamic neurons. Presented at the Annual Meeting of the Society for Neuroscience, New Orleans, LA. Abstract (no. 719.14).

Smith DM, Wakeman D, Patel J, Gabriel M. 2004. Fornix lesions impair context-related cingulothalamic neuronal patterns and concurrent discrimination learning. Behav Neurosci 118:1225-1239.

Song EY, Kim YB, Kim YH, Jung MW. 2005. Role of active movement in place-specific firing of hippocampal neurons. Hippocampus 15:8-17.

Sutherland RJ, Hoesing JM. 1993. Posterior cingulate cortex and spatial memory: a microlimnological analysis. In: Vogt BA, Gabriel M, editors. Neurobiology of Cingulate Cortex. Boston: Birkhauser. pp 461-477.

Sutherland RJ, Whishaw IQ, Kolb B. 1988. Contribution of cingulate cortex to two forms of spatial learning and memory. J Neurosci 8:1863-1872.

Tulving E. 2002. Episodic memory: From mind to brain. Ann Rev Psychol 53:1-25.

Vertes RP, Hoover WB, Viana Di Prisco G. 2004. Theta rhythm of the hippocampus: Subcortical control and functional significance. Behav Cogn Neurosci Rev 3:173-200.

Vinogradova OS. 1995. Expression, control, and probably functional significance of the neuronal theta-rhythm. Prog Neurobiol 45:523583.

Vogt BA, Miller MW. 1983. Cortical connections between rat cingulate cortex and visual, motor, and postsubicular cortices. J Comp Neurol 216:192-210.

Wagner AD, Shannon, BJ, Kahn R, Buckner RL. 2005. Parietal lobe contributions to episodic memory retrieval. Trends Cogn Sci 9:445-453.

Wilson MA, McNaughton BL. 1993. Dynamics of the hippocampal ensemble code for space. Science 261:1055-1058.

Wilson MA, McNaughton BL. 1994. Reactivation of hippocampal ensemble memories during sleep. Science 265:676-679.

Wood ER, Dudchenko PA, Eichenbaum H. 1999. The global record of memory in hippocampal neural activity. Nature 397:613-616.

Wood ER, Dudchenko PA, Tobitsek RJ, Eichenbaum H. 2000. Hippocampal neurons encode information about different types of memory episodes occurring in the same location. Neuron 27:623633.

Wyss JM, van Groen T. 1992. Connections between the retrosplenial cortex and the hippocampal formation in the rat: A review. Hippocampus 2:1-11.

Yeshenko O, Guazzelli A, Mizumori SJY. 2004. Context-dependent reorganization of spatial and movement representations by simultaneously recorded hippocampal and striatal neurons during performance of allocentric and egocentric tasks. Behav Neurosci 118: 751-769.

Zugaro MB, Monconduit L, Buzsáki G. 2005. Spike phase precession persists after transient intrahippocampal perturbation. Nat Neurosci $8: 67-71$. 